\title{
Amphotericin B: A New Look at Cellular Binding
}

\author{
John D. Cleary ${ }^{1, *}$ and Kishor M. Wasan ${ }^{2}$
}

\author{
${ }^{I}$ Department of Pharmacy Practice, Schools of Pharmacy \& Medicine, University of Mississippi Medical Center, 2500 \\ North State Street, Jackson, Mississippi 39216, USA \\ ${ }^{2}$ CIHR/iCo Therapeutics Research Chair in Drug Delivery for Neglected Global Diseases, Faculty of Pharmaceutical \\ Sciences, University of British Columbia, 2146 East Mall, Vancouver, British Columbia, Canada V6T 1Z3
}

\begin{abstract}
Objective: The binding characteristics of the polyene antifungal amphotericin B (AmB) are still arguably unclear 50 years after the first human treatment. Our purpose was to investigate the dynamic of polyene cell associated binding using an AmB directed antibody.

Methods: Ex vivo murine model and in vitro mammalian cells studies were utilized to assess the binding characteristics of $\mathrm{AmB}$ at human therapeutic concentrations. Balb/c mice administered a single dose of an AmB formulation were assessed using immunohistochemistry. Human mononuculear cells isolated after phlebotomy were assessed using transmission electron micrography.

Results \& Conclusions: Ex vivo studies demonstrated diffuse binding of AmB formulations, however, the sensitivity of the technique would not allow delineation of the antifungals' cellular binding. However, transmission electron micrography of in vitro cell cultures allowed apparent discrimination of cell associated binding and suggestions of intracellular trafficking within 2 hours of polyene exposure. One could hypothesize the observed characteristics and previously published literature are suggestive of binding to clathrin type receptors with internalization and processing by endosomes. Further work will be required to substantiate this hypothesis.
\end{abstract}

Keywords: Amphotericin B, cholesterol, binding, trafficking.

\section{INTRODUCTION}

Traditionally, the antifungal actions of amphotericin B [AmB] have been attributed to ergosterol binding to yeast and moulds. Polyenes like AmB have long been proposed to interact with mammalian cell membrane sterol (3-13hydroxy sterols), similar to ergosterol [1]. However, polyenes appear to bind with greater affinity to ergosterol containing membranes [2]. Both interactions have been reported to result in aqueous pore production. In unilamellar vesicles or cellular bilayers an annulus of eight $\mathrm{AmB}$ molecules can link hydrophobically to the membrane sterols [3]. The pore is configured with the polyene hydroxyl residues facing inward, leading to increased cell permeability, leakage of vital cytoplasmic components, and cell death [4-6]. Our purpose was to investigate the dynamic of polyene cell associated binding using an AmB directed antibody.

\section{MATERIALS AND METHODS}

Immunohistology and transmission electron microscope exploration of polyene cell associated binding were used in

*Address correspondence to this author at the Department of Pharmacy Practice, Schools of Pharmacy \& Medicine, University of Mississippi Medical Center, 2500 North State Street, Jackson, Mississippi 39216, USA; Tel: (601) 984-2617; Fax: (601) 984-2618;

E-mail: jcleary@umc.edu these trials. Polyene dynamics were assessed through secondary binding of a polyclonal antibody directed against AmB.

\section{REAGENTS}

\section{Amphotericin B Products and Controls}

The pharmacologic agents utilized included commercial grade AmB (Fungizone, Squibb; Princeton, NJ), liposomal AmB (Nexstar Pharmaceuticals, Boulder, CO; L-AmB), lipid complex AmB (Elan Pharmamceuticals, Bridgewater, $\mathrm{NJ}$; ABLC), AmB high purity (University of Mississippi Mycotic Research Center, AmBHP)[7]; Escherichia coli endotoxin (Serotype 026:b6 lipopolysaccharide, Sigma; St. Louis, MO) (LPS). Desoxycholate and sodium phosphate buffer were obtained from Sigma Chemical (St. Louis, MO) and empty liposomes from Nexstar Pharmaceuticals.

\section{Antibody}

A polyclonal rabbit antibody was isolated from New Zealand white rabbits after standard immunization with generic, desoxycholate solubilized AmB complexed with an immune adjuvant, Keyhole Limpet Hemocyanin. An anti$\mathrm{AmB}$ antibody was purified by filtering serum through an Aminolink Affinity-Pak Column (ImmunoPure $\mathrm{Ag} / \mathrm{Ab}$; Pierce Chemical Co., Rochford, IL) which was coupled with polyene. Column effluent was passed over a Protein G Column (Pierce Chemical Co., Rochford, IL) in order to separate immunoglobulin $\mathrm{G}$ ( $\mathrm{IgG}$ ) from immunoglobulin $\mathrm{M}$ 
(IgM). Evaluation of IgG purification process was validated utilizing a $7.5 \%$ SDS polyacrylamide gel. The final samples of anti-AmB IgG were frozen at $-70^{\circ} \mathrm{C}$ until further use [8].

\section{Gold Bead Labeling}

The procedure for colloidal gold conjugation antibodies has been described previously [9]. In brief, our method was modified in that a primary polyclonal rabbit antibody was used. Colloidal gold $8-12 \mathrm{~nm}$ particle diameter (Sigma; St. Louis, MO) was adjusted to $\mathrm{pH}=9$ with potassium acetate then conjugated with the antibody. An anti-AmB sample was diluted to a final protein concentration of $12 \mathrm{uM}$. This concentration was selected so there was a molar excess of antibody to "antigen". The ratio calculated was $4.4 \mathrm{x}$ based on each experimental well containing $27 \mathrm{uM}$ antibody.

\section{In Vitro Cell isolation and Preparation}

Heparinized blood was collected by peripheral venipuncture from normal healthy human volunteers. Institutional Review Board approval was obtained for collection of volunteer blood. No consent was required after assessment of minimal risk. Human mononuclear cell [MNC]s were sedimented in Mono-Poly Resolving Medium (Ficoll-Hypaque, Flow Laboratories; McLean, VA), removed by pipet and washed three times in unsupplemented media (RPMI - 1640; McLean, VA). MNCs were resuspended to a final concentration of approximately $5 \times 10^{6}$ cells $/ \mathrm{mL}$ in supplemented media (RPMI-1640, 10\% autologous serum, $100 \mathrm{ug} / \mathrm{mL}$ streptomycin and $100 \mathrm{u} / \mathrm{mL}$ penicillin). One milliliter of MNCs was seeded in Limbro 24 well plates (Flow Laboratories; McLean, VA) and incubated for one hour at $37^{\circ} \mathrm{C}$ in a $5 \% \mathrm{CO}_{2}$ prior to any study procedure. Initially, MNCs viability was assessed with tritiated $\left[{ }^{3} \mathrm{H}\right]$ thymidine $\left(1 \mu \mathrm{Ci} / 10^{6}\right.$ cells; $\left.62.5 \mathrm{pmol} / \mu \mathrm{Ci}\right)$ (Perkin Elmer, Boston, MA) incorporation was used to assess cytotoxicities of agents at varying concentrations and times of in vitro exposure (Fig. 1) and a nonspecific esterase stain was used to assess cell type. Aliquots of AmB solutions were added to cell culture wells, and the final concentrations of $\mathrm{AmB}$ preparations were obtained by addition of an equal volume of cell suspension. After establishing viability, the ability of MNCs to exclude trypan blue, as followed by light microscopy using routine procedures, was used to provide an assessment of cytotoxicity.

Average serum concentrations achieved in humans during FDA recommended AmB formulation dosing is $1.2 \mathrm{uM}$ for $\mathrm{AmB}, 83 \mathrm{uM}$ for Ambisome and $1.7 \mathrm{uM}$ for Abelcet. These concentrations were used in all in vitro exposure studies. High purity $\mathrm{AmB}$ has not undergone licensing trials at this time and no human serum concentrations are known. Therefore, serum concentrations achieved for generic amphotericin were used in AmBHP studies [7]. Reagents were diluted for in vitro studies with sterile water so that $0.01 \mathrm{~mL}$ of stock solutions added to culture wells resulted in the final concentrations noted. Samples from diluent (sterile water), media, reagents and flushed wells from culture plates were assayed for endotoxin utilizing the limulus amebocyte lysate. The limulus amebocyte lysate (Associates of Cape Cod Inc.; Woodshole, MA) testing employed an Escherichia coli endotoxin standard with a lower limit of detection of $3.0 \mathrm{pg} / \mathrm{mL}$. No

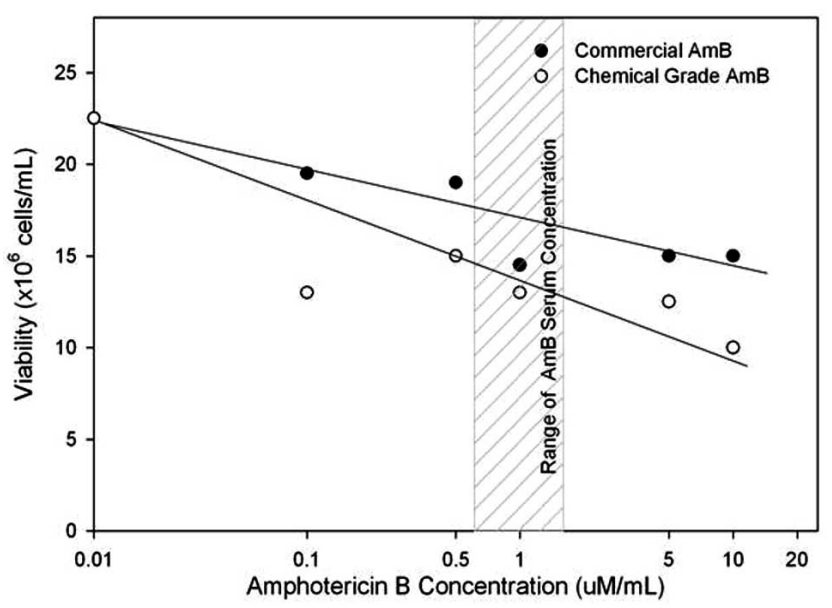

Fig. (1). Tritiated Thymidine Cytotoxicity Assay. Mononuclear cell viability was assessed for concentration and time dependency. In this figure, mononuclear cells were plated at $1 \times 10^{5}$ cells $/ \mathrm{mL}$ and exposed to varying polyene concentrations for 24 hours. Percent viability was determined from untreated control measured values. At exposures less than 24 hours, viability was improved for polyene concentrations greater than $1 \mathrm{uM}$.

reagents screened were positive. Media harvested from cellfree wells of culture plates were likewise negative.

\section{Immunohistochemistry for AmB}

In in vivo studies were performed after IACUC animal committee approval. One of the AmB formulations $(0.5 \mathrm{mg} / \mathrm{kg}$ of a non-lipid AmB formulation or $5 \mathrm{mg} / \mathrm{kg}$ of a lipid formulation) were administered intraperitoneally to fasting uninfected Balb/c mice over 2 minutes. These studies used AmB lipid complex as the only lipid formulation. At 24 hours the animals were sacrificed, vital organs (liver, spleen, liver \& lung) were isolated and transferred into $2 \mathrm{~mL}$ cold sterile phosphate-buffered saline. Samples were frozen at $80^{\circ} \mathrm{C}$ until analysis. Immunohistochemical staining was performed on routinely processed $4 \mu \mathrm{m}$ sections of paraffinembedded mouse tissue from animals treated with $\mathrm{AmB}$ formulations. Endogenous peroxidase was blocked in paraffin sections by immersing sections in $3 \% \mathrm{H}_{2} \mathrm{O}_{2} / \mathrm{PBS}$ for 30 minutes. Antigen retrieval was performed on paraffin sections by steam heating sections for 30 minutes in $\mathrm{Ag}$ Retrieval Citra-Plus ${ }^{\mathrm{TM}}$ buffer (Biogenex Corp., San Ramon, CA). Endogenous biotin was blocked using Biotin Blocking System TM (DAKO Corp, Carpinteria, CA). Anti-AmB antibody at a concentration of $\sim 3.5 \mu \mathrm{M}$ was applied on sections by incubating the section at $4^{\circ} \mathrm{C}$ overnight followed by biotinylated goat anti-rabbit $\operatorname{IgG}$ at $1: 1000$ and peroxidase conjugated streptavidin-biotin complex (Vector Lab. Inc, Burlingame, CA), according to the kit instruction. Briefly, after wash in PBS, sections were incubated with biotinylated secondary antibody for 30 minutes at room temperature. Following wash in PBS, the conjugate was added on sections, incubating at room temperature for 30 minutes, then washed sections in PBS again. Color was developed with 3,3'-diaminobenzidine (Vector Lab Inc), and sections were counterstained with hematoxylin Gill2 (Sigma, St. Louis, MO) and mounted in Cytoseal 60 (Steohens Scientific, Kalamazoo, MI). 
The negative controls consisted of sections of the liver, kidney, spleen and lung incubated with non-immune serum provided by Elite ABC kit (Verctor Lab.) instead of primary antibody. In addition, control, untreated mouse tissue were utilized. All staining performance was assessed in a double bind manner until interpretation complete.

\section{Transmission Electron Micrograph Study Procedure}

Human monocytes cultured at $5 \times 10^{6}$ cells $/ \mathrm{mL}$ were exposed to $\mathrm{AmB}$ for 5 minutes, 10 minutes, 20 minutes, 30 minutes, 1 hour, and 2 hours at either $4^{\circ} \mathrm{C}$ or $37^{\circ} \mathrm{C}$ in Coster 24 well culture plates. After exposure, cells were fixed. Visual identification of $\mathrm{AmB}$ was performed through complexing $\mathrm{AmB}$ in the cellular environment with an antibody bound to gold beads.

In pilot electron micrograph experiments, anti-AmB antibody bound to gold (AmBAg) was added to cultures 1 minute prior to fixing. These studies lead to micrograph studies that were un-interpretable. Washing and removing contaminating gold beads was difficult. In all further experiments, AmBAg was added after the cells were fixed, embedded and sliced for viewing. One milliliter of fixative (2.5\% glutaraldehyde solution mixed to a final concentration of $0.1 \mathrm{M}$ Sorensen's phosphate buffer) at $\mathrm{pH} 7.4$ was added to each $1.0 \mathrm{~mL}$ cell culture. After fixing, cells were embedded in $1 \%$ osmium, sliced and reacted with uranly acetate, dehydrated with ethanol, critical point dried then viewed.

\section{RESULTS}

Our in vivo immunohistologic studies investigated polyene distribution in tissue from treated animals. A sandwich assay, using a primary antibody against AmB and a secondary anti-rabbit antibody labeled with streptavidin biotin complex traced the polyene macroscopically. Diffuse binding was observed in these trials (Fig. 2). However, cellular features were difficult to discriminate and assessment of cellular dynamics was un-illustrative. More sensitive gold bead immuno-labeling performed in polyene exposed human mononuclear cell cultures revealed thought provoking results (Figs. $3 \&$ 4). In general, isolated human MNCs studied in vitro and examined by electron micrograph supported a new hypothesis of polyene action at routinely achievable serum concentrations.

The average cell yield from 90cc of human blood was $1.0-2.0 \times 10^{8}$ cells with greater than $95 \%$ viability (established by Trypan Blue exclusion \& tritiated thymidine). Viability was maintained at all 6 time points tested with exposure to AmB 1uM (Fig. 1). A nonspecific esterase test revealed an average of $66.3 \%( \pm 16.2)$ lymphocytes and $31.0 \%( \pm 11.7)$ monocytes. By trypan blue exclusion, AmB 5-10uM led to significant cell disruption and lysis. Studies with $\mathrm{AmB}$ greater than $5 \mathrm{uM}$ were not pursued and were deemed not clinically relevant. No affect on cell viability was observed with lipid formulation concentrations less than $10 \mathrm{uM}$.

\section{Immunohistochemistry for Amphotericin B}

Tissue sections appeared intact and revealed substantial staining for polyene treated sections compared to controls. In kidney sections, increased staining (increased brown color) was evident from $\mathrm{AmB}<\mathrm{AmBHP}<\mathrm{AmB}$ lipid complex compared to control (Fig. 2). AmB lipid complex differential staining compared to AmB should be associated with the higher dose of polyene. Similar staining was evident in liver, lung and spleen, however, less dramatic (data not shown). Unique to kidney samples, there appeared to be significantly more uptake in some renal tubular epithelial cells within the same section of tissue. In general, these data support the staining characteristics published previously [10]. Unfortunately, the magnification achievable with light microscopy does not allow discrimination of focal areas of staining.

\section{Transmission Electron Micrograph Study}

Even though an occasional damaged cell was evident, hypaq ficol isolation of mononuclear cells appeared robust. Cells were relatively round with some dendritic or areas of spreading (Figs. 3 \& 4). By 120 minutes, AmB stimulated cells appeared activated (fingers, dense granules, irregular borders, fingers or pseudopods, etc.). An occasional platelet was visible at 25,000 to 35,000 times magnification. Platelet contamination of the sample was unavoidable, yet, minimal.

Undamaged cells had significant AmB binding foci compared to control exposed cells at time points greater than 20 minutes. The majority of AmBAg accumulation appeared
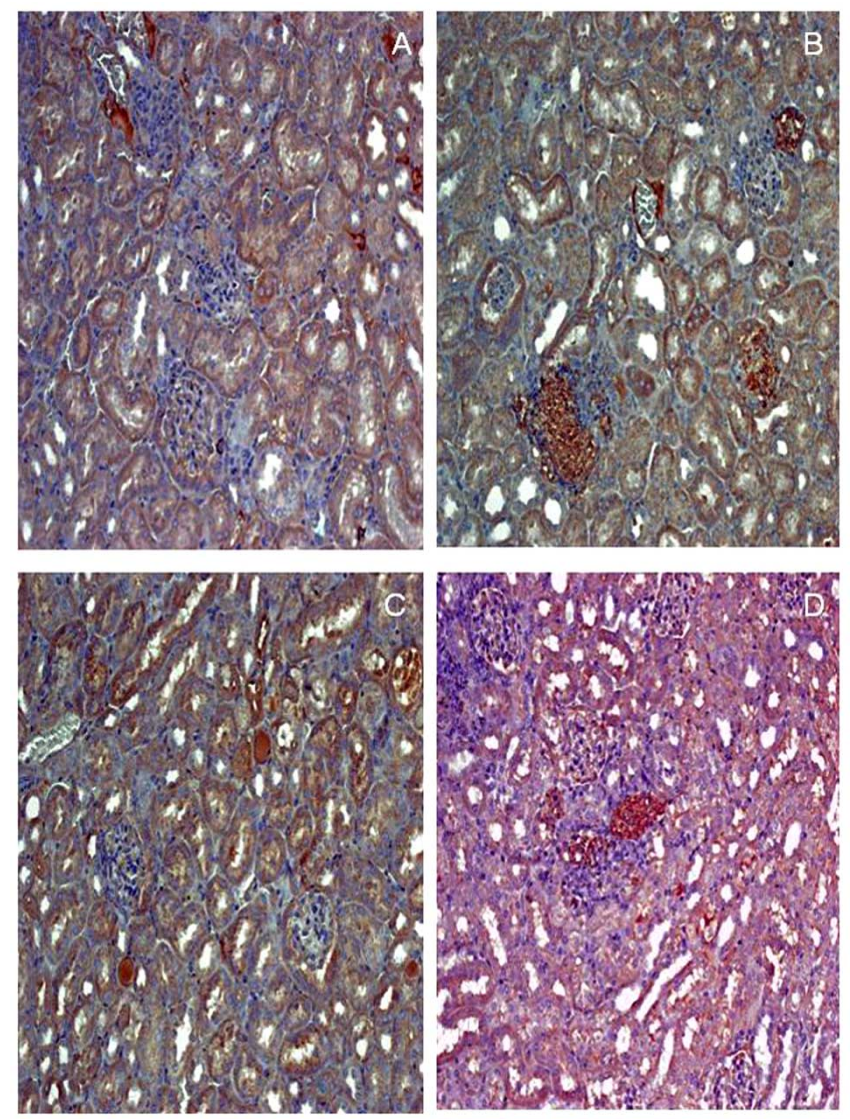

Fig. (2). Immunohistochemistry studies. Paraffin sections of mouse kidney after polyene treatment. Sections labeled by amphotericin B antibody then probed with biotinylated goat anti-rabbit $\operatorname{IgG}$ and peroxidase conjugated streptavidin-biotin complex. $\mathrm{A}=$ Amphotericin B $0.5 \mathrm{mg} / \mathrm{kg}, \mathrm{B}=\mathrm{AmBHP} 0.5 \mathrm{mg} / \mathrm{kg} \mathrm{C}=\mathrm{ABLC} 5 \mathrm{mg} / \mathrm{kg}$ $\mathrm{D}=$ Control. Magnification 20X. 
to be within localized points or vesicles which look similar to lysosomes. We suggest that the vesicles or bodies are digestive vacuoles or dense granules that occur in white blood cells. Accumulation or collection of AmBAg does not appear to be dependent on time. There are not vesicles with large numbers of AmBAg, nor are the large quantities free in the cytoplasm or attached to the cell membrane. However, due to the known rapid cycling of these vesicles, one could hypothesize that our cross sections of time captured did not allow full elucidation of the cycle.
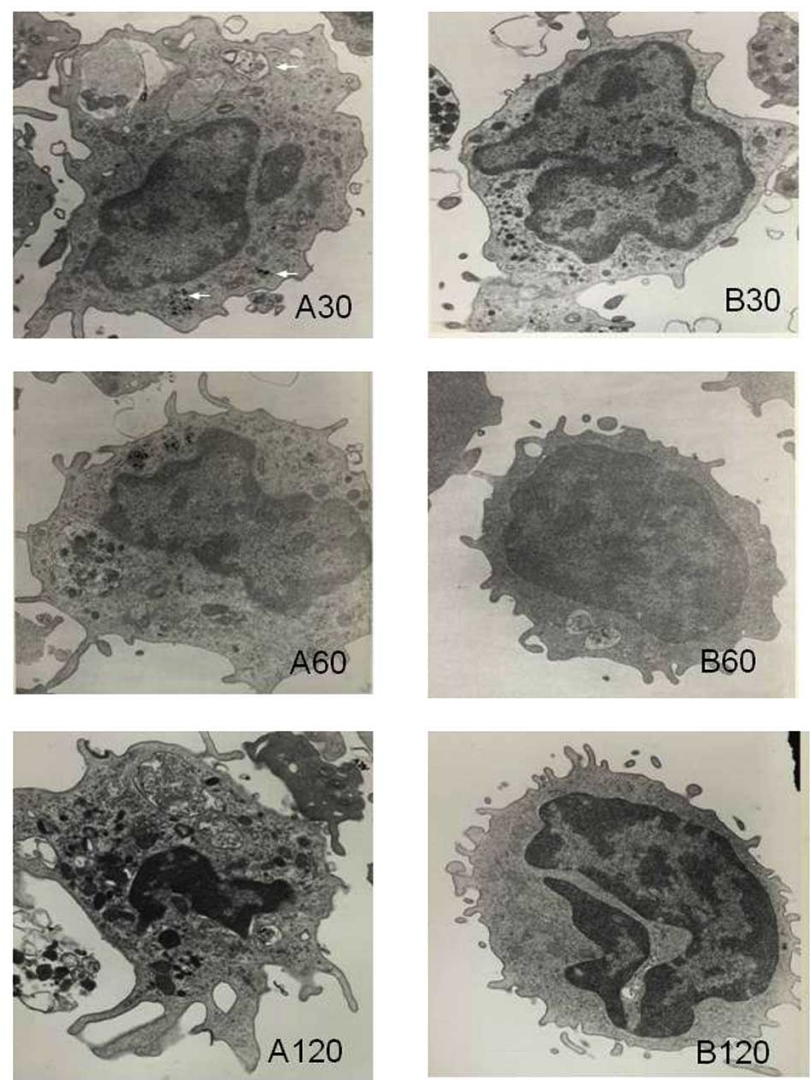

Fig. (3). Transmission electron micrograph studies. Experimental conditions (A) and control (B) are shown for 30 minutes (30), 60 minutes (60), and 120 minutes (120). Magnification of images are $\mathrm{x} 25,000$.
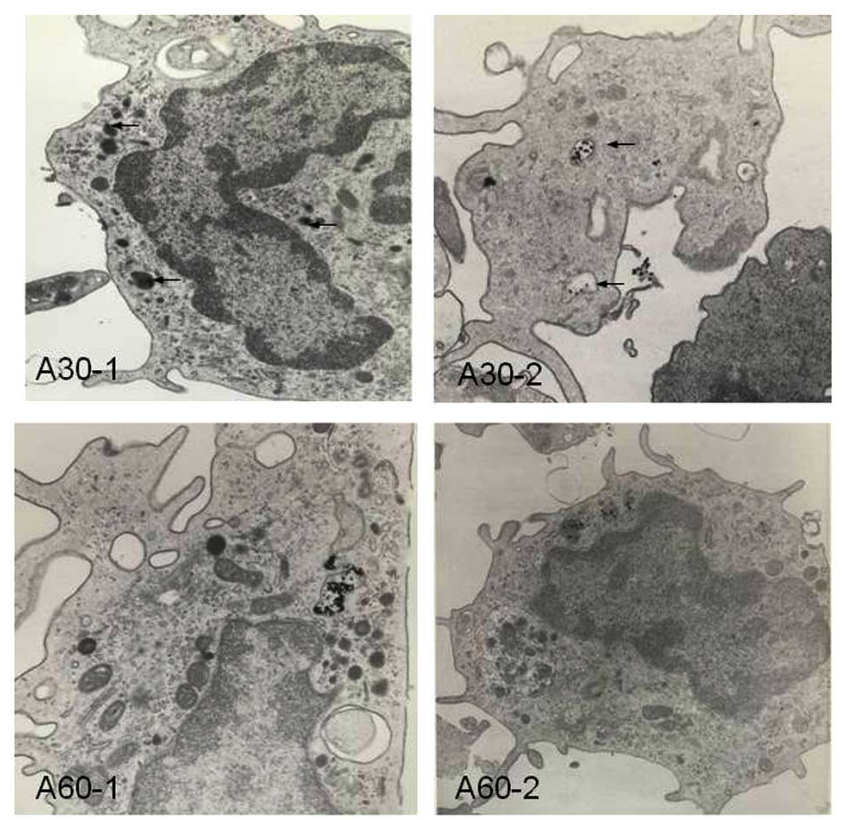

Fig. (4). Transmission electron micrograph studies. Exposure to amphotericin B for 30 and 60 minutes at $x 35,000$. Digestive vacuoles are clearly evident at this magnification. An arrow signifies digestive vacuoles or alpha granules. Notice double membrane, unlike a dense granule (serotonin does not appear evident by color.

Since cholesterol is abundant on mammalian cells, one could expect random binding of $\mathrm{AmB}$ to cell surfaces. One can observe that $\mathrm{AmB}$ binding does not appear to be random. Intact cell membranes can be observed without any visible AmbAg complexes, yet, one can observe clusters of drug in localized points. Binding characteristics of $\mathrm{L}-\mathrm{AmB}$ and ABLC exposure lead to identical electronic micrograph observations (data not shown). Based on the biologic assessments that phagocytic cells internalize $100 \%$ of their plasma membrane every few hours, one might expect to also find substantial internalized cell membrane or free AmB intracellularly if binding was random to cell wall cholesterol. However, none were visible. Alternatively, at very high LDL

\section{CLATHRIN OR CAVEOLIN COATED PITS - INTERACTIONS WITH AMPHOTERICIN}

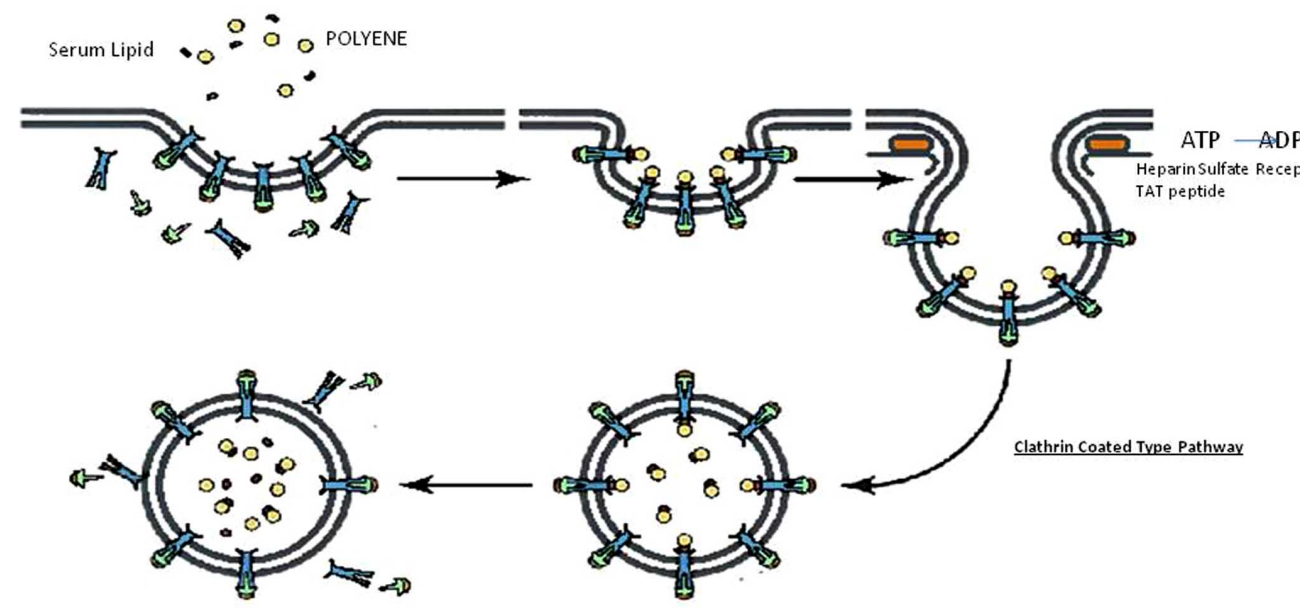

Fig. (5). A hypothesized model of amphotericin b's interaction with a mammalian cell at concentrations routinely found in the serum. 
concentrations, LDL binds to specific coated pits (concave clathrin coated areas of cell membrane) and known to be internalized into endosomes after short periods. The biology of hormones support the electron micrograph observations.

\section{DISCUSSION}

It is clear that $\mathrm{AmB}$ binds to multiple tissues. Autopsy studies evaluating drug penetration suggest that adipose is the only tissue without significant quantities of AmB [11]. Immuno-histochemistry, as a tool for tracking drug movement revealed AmB binding to kidney, liver, lung and spleen (Fig. 2). Similar studies have been performed demonstrating preferential binding after several days of therapy in spleen, then liver, kidney and lung [10]. Immunopositive staining was evident on the cytoplasm of Kupfer cells. Due to the sensitivity of staining, it was difficult to discern anatomical intracellular binding sites on tissue. One could conclude that binding appeared on and in cells tested.

Transmission micrograph revealed AmB clusters on the cell's surface. These clusters appear to be in "pits" that could lead one to hypothesize that binding is not diffuse. In one image (Fig. 4; A30-2), one can visualize the leading edge of a leukocyte with indentations (pits) filled with AmBAg. Based on known AmB binding to lipoproteins, one could also hypothesize these pits are the clarthrin coated or lipid receptors. Generally, the time required to process a ligand can range 5 minutes or longer. Once LDL receptors are internalized, endocytic vesicles contain $\sim 6 \%$ clathrin by volume, or $\sim 8 \%$ of the wall surface area. Kinetic studies have long shown that these receptors recycle back to the cell surface at a half-life of $\sim 5$ minutes [12]. Since the process is dyanamic and with excess AmB relative to "receptors", our experiments may have captured mature receptor + ligand $(\mathrm{AmB}+$ Lipoprotein) processing in motion (Fig. 5). Literature to support this hypothesis includes years of lipoprotein research.

Brajtburg and coworkers initially examined the interactions of $\mathrm{AmB}$ with human serum lipoproteins in vitro in an attempt to understand these interactions and how they might affect the pharmacological behavior of AmB. Their studies showed AmB to be equally associated with highdensity lipoprotein (HDL) and low-density lipoprotein (LDL) fractions after 1 hour of incubation at $25^{\circ} \mathrm{C}$ [13]. Furthermore, $\mathrm{AmB}$ and $\mathrm{LDL}$ mixtures injected into rabbits was toxic: $70 \%$ of the rabbits died from a non-toxic dose of AmB $(1.0 \mathrm{mg} / \mathrm{kg})$, which implies that LDL association would increase AmB cellular uptake/binding and toxicity [13].

These same investigators previously demonstrated that HDL-associated AmB 20uM and HDL-associated L-AmB $20 \mathrm{uM}$ are less toxic to LLC PK1 renal cells than are AmB or LDL-associated AmB. The reduced toxicity of HDLassociated AmB may be explained by the low level of expression of HDL receptors in LLC PK1 cells. The sustained toxicity observed with $\mathrm{AmB}$ alone in trypsinized cells may be related to a direct membrane effect. However, when $\mathrm{AmB}$ is associated with LDL, the toxicity is maintained, which suggests that both direct membrane- and non-membrane-related toxicity may occur. Furthermore, a study to determine if a relationship existed between serum lipoprotein cholesterol concentration and the severity of
AmB-induced renal toxicity in patients suggested that patients with higher serum LDL-cholesterol concentrations are more susceptible to AmB-induced renal toxicity [13]. Interestingly, $\mathrm{AmB}$ complexed with lipid is less toxic than the self associated form of $\mathrm{AmB}$ in medium, but the monomeric form of $\mathrm{AmB}$ interacts with fungal cells membrane and is non-toxic against mammalian cells membrane [14].

The results we obtained previously demonstrated that changes in temperature and association with lipid-complexes affect the distribution of $\mathrm{AmB}$ in serum lipoproteins [13]. Similar to the results reported by Brajtburg et al., when serum lipoproteins were separated by affinity chromatography, an equal distribution of $\mathrm{AmB}$ was found in human serum lipoprotein fractions following one-hour incubation at $25^{\circ} \mathrm{C}$ [15]. In contrast, at $37^{\circ} \mathrm{C}$, over $90 \%$ of the concentration of $\mathrm{AmB}$ was found in the HDL fraction following 1 hour incubation. However, recent studies in our lab using density gradient ultracentrifugation to separate plasma lipoproteins suggest this is an overestimation of the percentage of AmB recovered in the HDL fraction. Between $66-80 \%$ of the original concentration incubated in plasma was recovered in the lipoprotein-deficient plasma fraction, which contains mainly albumin and B-1-glycoprotein. This inconsistency in results is due to the limitation of the affinity chromatography technique in determining the lipoprotein distribution of drugs. This technique can only separate LDL and VLDL away from the other plasma proteins and lipoproteins. It cannot separate HDL from the plasma proteins (albumin and B-1-glycoprotein). Using densitygradient ultracentrifugation, the different lipoproteins can be separated away from plasma proteins. The core of HDL becomes more ordered at the higher temperature, thus making it easier for the $\mathrm{AmB}$ molecule to associate with it. This hypothesis is based on the assumption that AmB is incorporated into the lipophilic core of these lipoproteins. A final argument for the proposed hypothesis has been the affects of LDL. Studies that have blocked the LDL receptor by pre-incubating $\mathrm{CHO}$ cells with trioleoyl glycerol triolein bound LDL demonstrated AmB associated cellular toxicity is abolished [16].

Transmission electron micrograph studies in lipid research reveal support for our proposed hypothesis. When human umbilical artery endothelial cells were cultured for 18 hours in LDL depleted serum, to increase LDL receptor density, LDL bound to gold beads could be found in large vesicles with an electron-lucent matrix and smooth membranes (endosomes) [17]. In these studies, gold labeled LDL (30-50 ug/protein $/ \mathrm{mL}$ ) was incubated for 5,15 , and 30 minutes at $37^{\circ} \mathrm{C}$ with cells and then washed. By 30 minutes, endosomes could be found that contained electron-dense particles close to endosome membranes. After short periods, internalization of LDL into endosomes had already started. These authors suggest that the LDL plus gold is too large to undergo pinocytosis (low-affinity pathway). They hypothesized that, since LDL delivery and degradation of LDL had been established in the literature, it may be reasonable to assume the dense bodies are lysosomes containing degraded LDL and the gold-antibody.

Kinsky et al., was one of the first to describe transmission electron micrograph changes associated with a 
polyene [18]. These researchers were using a polyene to track cholesterol. They described pits $\left(200-250 \mathrm{~A}^{\circ}\right)$ in rat erythrocytes formed by filipin. Pit formation appeared to be dependent on cholesterol content. They hypothesized the formed pits were a sign of membrane destabilization. However, another interpretation of these observations could lead one to conclude that cholesterol (LDL) bound polyene attach in those clathrin coated pits. Filipin, a polyene, binds specifically to cholesterol and has also had widespread use as a histiochemical fluorescent stain. Yet, filipin is a pentaene antibiotic which does not contain a carboxyl group or mycosamine sugar moiety and the affinity of filipin to cholesterol is stronger than to ergosterol $[6,19]$.

Experiments evaluating cellular quantities of $\mathrm{AmB}$ have lead to varying estimates, yet are insightful. Subtractive absorption spectrophotometry using a fluorescence probe (1[4-(trimethylammonio)phenyl]-6-phenylhexa-1,3,5-triene, $\mathrm{p}$ toluenesulfonate [TMA-DPH]) was utilized to evaluate polyene binding in $\mathrm{L}$ cells. The polyene surface density obtained was $3.7 \times 10^{-3} \mathrm{AmB}$ per lipid on the cell surface or approximately $1 \mathrm{AmB}: 270$ lipids, or $1.6 \%$ of $\mathrm{AmB}$ added at 2 uM [20]. Hoessli \& Runger-Brandle estimated that murine T-lymphocytes (where cholesterol=phospholipids) have $3.4 \times 10^{9}$ plasma membrane lipids. Mathematically, one could estimate an absurd $1.25 \times 10^{7} \mathrm{M} \mathrm{AmB}$ per cell using the above estimates. More plausible estimates are obtained by other methodologies. Determination of monocyte associated drug by scanning spectrophotometry (350 to $450 \mathrm{~nm}$ ) was performed using overnight co-incubation, lysing and assay of intracellular fluid assayed. The absorbance difference between the trough at $398 \mathrm{~nm}$ and the peak at $408 \mathrm{~nm}$ was determined. Incubation with $\mathrm{AmB} 1.0 \mathrm{uM}$ resulted in intracellular concentrations, as early as 30 minutes that progressively increased over 20 hours to a maximum of $\sim 50 \mathrm{fg} /$ cell or an estimated intracellular concentration of $\sim 100$ pM (assuming a cell volume of $500 \mathrm{fL}$ per cell) [21]. Phagocytic cells have been shown to accumulate AmB 0.8 \& $3.5 \mathrm{ng} / 10^{6}$ peritoneal exudative cell and L-AmB 4.4 \& $7.9 \mathrm{ng} / 10^{6}$ cells in vivo at 6 and 24 hours after AmB administration [22]. Performing a similar calculation, they are estimating $160 \mathrm{pM}$ to $7000 \mathrm{pM}$ and $8800 \mathrm{pM}$ to $15,800 \mathrm{pM}$ for $\mathrm{AmB}$ and a liposomal formulation. Combined, these studies support that therapeutic concentrations of AmB do not bind to all cellular associated cholesterol.

Recent studies on cholesterol regulation suggests ABCA1 localizes to the plasma membrane and is functional in the efflux of excess cellular cholesterol into high-density lipoprotein particles. The sub-cellular localization of the ABCA2 transporter in lysosomes suggested a possible role for $\mathrm{ABCA} 2$ in regulating the movement or efflux of LDL from the lysosme to the plasma membrane and endoplasmic reticulum [23]. Our pharmacogenomic data support upregulation of transcripts (ABCA 2, LDLR, HMGCoA S AND SREBP2) associated with the lipid export machinery during AmB exposure. These suggest that lipid bound AmB may activate a signal transduction pathway in mononuclear cells $[24,25]$.

\section{CONCLUSION}

Significant binding of AmB to low density lipoproteins, accumulation of $\mathrm{AmB}$ within white blood cell vesicles, digestive vacuoles or dense granules supports a new hypothesis for polyene interaction with human cells. We propose an alternate hypothesis of polyene binding. AmB, at pharmacotherapeutically achievable serum concentrations, binds preferentially to soluble serum lipoproteins. We suggest that this $\mathrm{AmB}$ - cholesterol ligand is collected into clathrin coated pits and enters into intercellular trafficking and signaling. Previous literature supports signaling activation and secondary cellular protein expression (i.e. inflammatory cytokines, apoptosis) [26]. What is unclear are the events transpiring in the digestive vacuoles leading to protein expression. It is known that primary lysosomes (25$50 \mathrm{~nm})$ empty their contents into vacuoles to digest their contents. These secondary lysosome $(<400 \mathrm{~nm})$ or endosomes dump their digested contents back into the extracellular space [12]. Continued exploration of this hypothesis will be required to elucidate the fate of endocytosed AmB.

\section{TRIAL REGISTRATION}

None Required.

\section{FUNDING}

These studies were supported by the University of Mississippi in kind.

\section{TRANSPARENCY DECLARATION}

Neither J.C. or K.W. have received funds for speaking or research from pharmaceutical companies related to this work. J.C. is a holder of the amphotericin B High Purity Patent.

\section{CONFLICT OF INTEREST}

None declared.

\section{ACKNOWLEDGEMENTS}

None.

\section{REFERENCES}

[1] Gruda I, Nadeau P, Brajtburg J, et al. Application of different spectra in the UV-visible region to study the formation of amphotericin B complexes. Biochim Biophys Acta 1980; 602: 260-8.

[2] Vertut-Croquin A, Bolard AJ, Chabert M, et al. Differences in the interaction of the polyene antibiotic amphotericin B with cholesterol- or ergosterol-containing phospholipids vesicles. A circular dichroism and permeability study. Biochemistry 1983; 22: 2939-44.

[3] Cheron M, Cybulska B, Mazerski J, et al. Quantitative structureactivity relationships in amphotericin B derivatives. Biochem Pharmacol 1988; 37: 827-36.

[4] Kotler-Brajtburg J, Medoff G, Kobayashi GS, et al. Classification of polyene antibiotics according to chemical structure and biological effects. Antimicrob Agents Chemother 1979; 15: 71622.

[5] Urbina JA, Cohen BE, Perozo E, et al. Spin-labeled amphotericin B: synthesis, characterization, biological and spectroscopic properties. Biochim Biophys Acta 1987; 897: 467-73.

[6] Bolard J. How do the polyene macrolide antibiotics affect the cellular membrane properties? Biochim BiophyActa 1986; 864: 257-304.

[7] Cleary JD, Chapman SW, Swiatlo E, et al. High purity amphotericin B. J Antimicrob Chemother 2007; 60: 1331-40.

[8] Cleary JD, Chapman SW, Deng J, et al. Amphotericin B Enzyme Linked Immunoassay. Antimicrob Agents Chemother 1996; 40: 637-41. 
[9] Kramarcy NR, Sealock R. Commercial preparations of colloidal gold-antibody complexes frequently contain free active antibody. J Histochem Cytochem 1991; 39(1): 37-9.

[10] Smith PJ, Olson JA, Constable D, et al. Effects of dosing regimen on accumulation, retention and prophylactic efficacy of liposomal amphotericin B. J Antimicrob Chemother 2007; 59: 941-51.

[11] Christiansen KJ, Bernard EM, Gold JW, et al. Distribution and activity of amphotericin in humans. J Infect Dis 1985; 152: 1037.

[12] Gruenberg J, Howell KE. Membrane traffic in endocytosis: insights from cell free assays. Annu Rev Cell Biol 1989; 5: 453-81.

[13] Wasan KM, Brocks DR, Lee SD, et al. Impact of lipoproteins on the biological activity and disposition of hydrophobic drugs: implications for drug discovery. Nat Rev Drug Discov 2008; 7: 84-99.

[14] Bolard J, Legrand P, Heitz F, et al. One-sided action of amphotericin B on cholesterol-containing membranes is determined by its self-association in the medium. Biochemistry 1991; 30: 5707-15.

[15] Brajtburg J, Elberg S, Bolard J, et al. Interaction of plasma proteins and lipoproteins with amphotericin B. J Infect Dis 1984; 149: 986-97.

[16] Krieger M, Martin J, Segal M, et al. Amphotericin B selection of mutant Chinese hamster cells with defect in the receptor-mediated endocytosis of low density lipoprotein and cholesterol biosynthesis. Proc Natl Acad Sci 1983; 80: 5607-11.

[17] Mommaas-Kienhuis AM, Krijbolder LH, van Hinsbergh VWM, et al. Visualization of binding and receptormediated uptake of low density lipoproteins by human endothelial cells. Eur J Cell Biol 1985; 36: 201-8

[18] Kinsky SC, Luse SA, Zopf D, et al. Interaction of filipin and derivatives with erythrocyte membranes and lipid dispersions: electron microscopic observations. Biochim Biophys Acta 1967; 135: 844-61.

[19] Wasan KM, Lopez-Berestein G. Diversity of lipid-based polyene formulations and their behavior in biological systems. Eur J Clin Microbiol Infect Dis 1997; 16: 81-92.

[20] Henry-Toulme N, Seman M, Bolard J. Interaction of amphotericin $\mathrm{B}$ and its $\mathrm{N}$-fructosyl derivative with murine thymocytes: a comparative study using fluorescent membrane probes. Biochim Biophys Acta 1989; 982: 245-52.

[21] Martin E, Stüben A, Görz A, et al. Novel aspect of amphotericin B action: accumulation in human monocytes potentiates killing of phagocytosed Candida albicans. Antimicrob Agents Chemother 1994; 38: 13-22.

[22] Mehta RT, McQueen TJ, Keyhani A, et al. Phagocyte transport as mechanism for enhanced therapeutic activity of liposomal amphotericin B. Chemotherapy 1994; 40: 256-64.

[23] Davis W Jr, Boyd JT, Ile KE, et al. Human ATP-binding cassette transporter-2 (ABCA2) positively regulates low-density lipoprotein receptor expression and negatively regulates cholesterol esterification in Chinese hamster ovary cells. Biochim Biophys Acta 2004; 1683: 89-100.

[24] Cleary JD, Deng J, Cao W, et al. Altered gene expression with Amphotericin B Exposure. In: Proceedings of the 36th Annual Interscience Conference on Antimicrobial Agents and Chemotherapy Meeting 1996; 324.

[25] Gumbo T, Cleary J. In: Zdanosicz M, Ed. Concepts in Pharmacogenomics. First Edition. Bethesda, American Society of Healthsystems Pharmacists, Inc. 2009, 365-82.

[26] Cleary JD, Nolan R, Chapman SW. Inhibition of interleukin 1 release from endotoxin or amphotericin B stimulated monocytes. Antimicrobial Agents Chemother 1992; 36: 977-81.

(C) Cleary and Wasan; Licensee Bentham Open.

This is an open access article licensed under the terms of the Creative Commons Attribution Non-Commercial License (http://creativecommons.org/licenses/ by-nc/3.0/) which permits unrestricted, non-commercial use, distribution and reproduction in any medium, provided the work is properly cited. 